\title{
Long-Term Follow-Up of Autonomic and Enteric Measures in Patients Undergoing Vertical Banded Gastroplasty for Morbid Obesity
}

\author{
Neil E. Crittenden ${ }^{\mathrm{a}}$, Hani Rashed ${ }^{\mathrm{b}}$, William D. Johnson ${ }^{\mathrm{c}}$, George Cowan ${ }^{\mathrm{b}}$, David Tichansky ${ }^{\mathrm{d}}$, \\ Atul Madan ${ }^{\mathrm{e}}$, Naeem Aslamb ${ }^{\mathrm{b}}$, Teresa Cutts ${ }^{\mathrm{f}}$, Thomas L. Abell ${ }^{\mathrm{a}, \mathrm{g}}$
}

\begin{abstract}
Background: A multi-component model of autonomic and enteric factors may correlate with ultimate weight loss or gain after restrictive obesity surgery. This study aimed to determine relevant parameters to predict successful long-term weight loss.

Methods: Thirty-nine patients (four males and 35 females) with a mean age of 37.2 years were followed for over 15 years after vertical banded gastroplasty. Baseline adrenergic: postural adjustment ratio (PAR) and vasoconstriction (VC); cholinergic: electrocardiogram Rto-R interval (RRI) and enteric measure: electrogastrogram (EGG) were utilized by a discriminant function analysis to classify patients as a long-term loser or gainer. Using latest weight compared to baseline, patients were divided as 10 gainers and 29 losers.
\end{abstract}

Results: A discriminate model successfully predicted ultimate weight gain in $8 / 10(80 \%)$ of patients who subsequently gained weight and weight loss in $24 / 29(83 \%)$ of patients who lost weight for a total correct classification of 32/39 (82\%). The same model with data at 3 months postoperatively predicted weight gain in 9/10 (90\%) of patients and weight loss in $24 / 29(83 \%)$ of patients, for a total correct classification of $34 / 39(87 \%)$.

Conclusions: A multi-component model at baseline and 3 months postoperative can predict long-term weight outcome from restrictive obesity surgery.

Keywords: Obesity; Vertical banded gastroplasty; Autonomic nervous system; Eenteric nervous system

Manuscript submitted July 17, 2017, accepted August 23, 2017

aUniversity of Louisville, Louisville, KY, USA

bUniversity of Tennessee Health Science Center and Methodist Hospital, Memphis, TN, USA

'Pennington Biomedical Research Center, Baton Rouge, LA, USA

dJefferson University, Philadelphia, PA, USA

${ }^{\mathrm{e}}$ Marina del Ray Hospital, Los Angeles, CA, USA

fWake Forest University, Winston-Salem, NC, USA

gCorresponding Author: Thomas Abell, Division of Gastroenterology, Hepatology and Nutrition, University of Louisville, 550 S Jackson Street, ACB3 A3L15, Louisville, KY 40202, USA. Email: thomas.abell@louisville.edu

doi: https://doi.org/10.14740/gr885w

\section{Introduction}

No current biologic marker exists for sub-classification of obesity in terms of outcome of therapies. Part of the pathophysiology of severe obesity may involve the autonomic nervous system (ANS) [1, 2]; previous reports have also implicated enteric abnormalities [3]. In other severe medical conditions involving the ANS and enteric nervous system (ENS), physiologic testing has been useful to predict therapeutic outcomes [4-6]. Vagal sensory activity has been implicated as part of the hypothalamic and cortico-limbic control helping to regulate energy balance [7]. This mechanism was explored in clinical trials for intra-abdominal vagal nerve blockade for the treatment of obesity [8]. The vagal nerve is part of the ANS which was non-invasively measured by vasoconstriction (VC), postural adjustment ratio (PAR) and R-to-R interval (RRI) in our study. The ENS was measured using cutaneous electrogastrogram (EGG).

The availability of ANS and ENS testing has been important in the identification of specific abnormalities in illnesses with severe organ involvement or failure $[6,9,10]$. Based on the evaluation of organ failure patients with ANS/ENS testing, we hypothesized that the results might be abnormal in patients with severe obesity.

It has been previously reported that both ANS measures at 1 - 2 years and ENS measures at 4 - 5 years may be helpful in predicting long-term outcomes of surgical therapies for obesity [11-19]. This study aimed to determine whether an ANS/ ENS-based model at baseline and at 3 months postoperatively predicts long-term weight outcome in morbidly obese patients undergoing vertical banded gastroplasty.

\section{Materials and Methods}

Thirty-nine morbidly obese patients were studied between 1988 and 1990 before undergoing a restrictive operation - vertical banded gastroplasty - for severe obesity [20, 21]. Sample size was the number of patients who volunteered for the study. Patients were four males and 35 females, with a mean age of 37 years, and all had a medical evaluation prior to surgery. Mean baseline weight for all 39 patients was 292 lbs; weights were reported as absolute weight, rather than as body mass 
Table 1. Participants and Individual Autonomic Measurements

\begin{tabular}{|c|c|c|c|c|c|c|c|c|c|c|}
\hline \multirow[b]{2}{*}{ Parameter } & \multirow{2}{*}{$\begin{array}{l}\text { Normal } \\
\text { value }\end{array}$} & \multicolumn{3}{|c|}{ Student's $t$-test of means } & \multicolumn{3}{|c|}{ Actual weight gainers $(n=10)$} & \multicolumn{3}{|c|}{ Actual weight losers $(n=29)$} \\
\hline & & $\begin{array}{l}\text { Gainers } \\
(n=10)\end{array}$ & $\begin{array}{l}\text { Losers } \\
(n=29)\end{array}$ & P-value & $\begin{array}{l}\text { n } \\
\text { normal }\end{array}$ & $\begin{array}{l}\text { n } \\
\text { abnormal }\end{array}$ & $\begin{array}{l}\% \\
\text { abnormal }\end{array}$ & $\begin{array}{l}\text { n } \\
\text { normal }\end{array}$ & $\begin{array}{l}\text { n } \\
\text { abnormal }\end{array}$ & $\begin{array}{l}\% \\
\text { abnormal }\end{array}$ \\
\hline Mean age at surgery (years) & & 32.00 & 39.04 & 0.0409 & & & & & & \\
\hline Mean weight at surgery (lbs) & & 309.30 & 289.34 & 0.3615 & & & & & & \\
\hline Mean PAR (\%) & $>25$ & 35.73 & 51.66 & 0.11 & 8 & 2 & $20 \%$ & 20 & 9 & $31 \%$ \\
\hline Mean RRI (\%) & $>33$ & 30.90 & 24.61 & 0.21 & 5 & 5 & $50 \%$ & 8 & 21 & $72 \%$ \\
\hline
\end{tabular}

EGG: electrogastrogram; VC: vasoconstriction; PAR: postural adjustment ratio; RRI: R-to-R interval.

index (BMI) or percent excess weight. There was no difference in the mean weight at surgery or the percentage of females; however, the gainers were statistically significantly younger at a mean age of 32 years compared to a mean age of 39 years for the eventual weight losers $(\mathrm{P}=0.0409)$. All patients underwent baseline ANS and ENS assessments prior to vertical banded gastroplasty in the years 1989 - 1992 (Table 1). The patients were subsequently followed for assessment of current weight. We examined over 15 years of follow-up data to investigate which relevant parameters might predict successful long-term weight loss.

Patients who elected to have vertical banded gastroplasty underwent autonomic testing and their postoperative weights were collected over a period of 3 - 15 years with an attempt to follow the patients for up to 25 years. During the study period, they received routine care.

Autonomic testing was performed prior to surgery by measuring adrenergic, cholinergic and enteric variables. The adrenergic nervous system was measured using the PAR and $\mathrm{VC}$ and expressed as a percentage. The cholinergic nervous system was measured using the electrocardiogram (EKG) RRI [2] and also expressed as a percentage [14, 22]. Gastric electrical activity was also measured by an electrogastrogram (EGG) and expressed in cycles per minute (cpm) with a normal value being less than $3.3 \mathrm{cpm}$ [22-25].

Adrenergic nervous system evaluation was based on capillary photoplethysmography which measures peripheral blood flow at rest, in response to postural maneuvers and cold stress. This system has been validated by catecholamine level [26]. Cholinergic function was measured by cardiac (EKG) RRIs, in response to deep breathing and Valsalva maneuvers. ENS function was measured by cutaneous electrogastrography and was analyzed by signal averaging; both techniques have been used previously [27].

Investigators following the weight changes postoperatively were blinded to the preoperative autonomic testing results. Investigators reviewed the weight changes of individual subjects and determined if the subjects were long-term weight gainers or losers. Weight gainers were defined as patients who resumed inappropriate weight gain after an early rapid weight loss (Supplementary 1, www.gastrores.org). Weight losers continued long-term postoperative weight loss or achieved and maintained a healthy long-term body weight.

After subjects were stratified between those with continued weight gain and those with continued weight loss, the autonomic testing values were analyzed to develop a multivariate discriminate model whereby a criterion was determined from the autonomic testing to discriminate the long-term weight gainers from the long-term successful losers. The performance of the discriminant criterion was evaluated in terms of how well the model correctly classified the true gainers as gainer, as well as how many losers were correctly classified as loser.

The statistical analysis was a discriminant function analysis which can predict categorical dependent variable (in our case weight gain) by multiple independent predictor variables. In this application, the predictor variables are the various autonomic parameters clinically measured: PAR, RRI, VC, and EGG. The analysis created linear combinations of predictors and by doing so created a new latent variable for each function. Successive functions are created that maximize difference on that function, and must not be correlated with the previous function created.

Repeated attempts to obtain additional weight loss data beyond approximately 15 years did not result in a measurable number of new data points, even when patient data were available for review up to 25 years post-surgery.

\section{Results}

A committee blinded to the preoperative autonomic testing results evaluated each patient and grouped them into long-term actual weight gainers $(\mathrm{n}=10)$ and long-term weight losers $(\mathrm{n}=$ 29) based on absolute weight loss and long-term changes over time. A weight loser either regains less than 20 pounds after reaching a nadir weight of less than 300 pounds or maintains a final weight less than 175 pounds. A weight gainer never reaches a nadir weight of less than 300 pounds, regains more than 20 pounds or attains a final weight more than 175 pounds (Fig. 1) (Supplementary 1, www.gastrores.org).

A discriminate model based on baseline measures successfully predicted ultimate weight gain in $8 / 10(80 \%)$ of patients who subsequently gained weight and weight loss in 24/29 (83\%) of patients who subsequently lost weight for a total correct clas- 


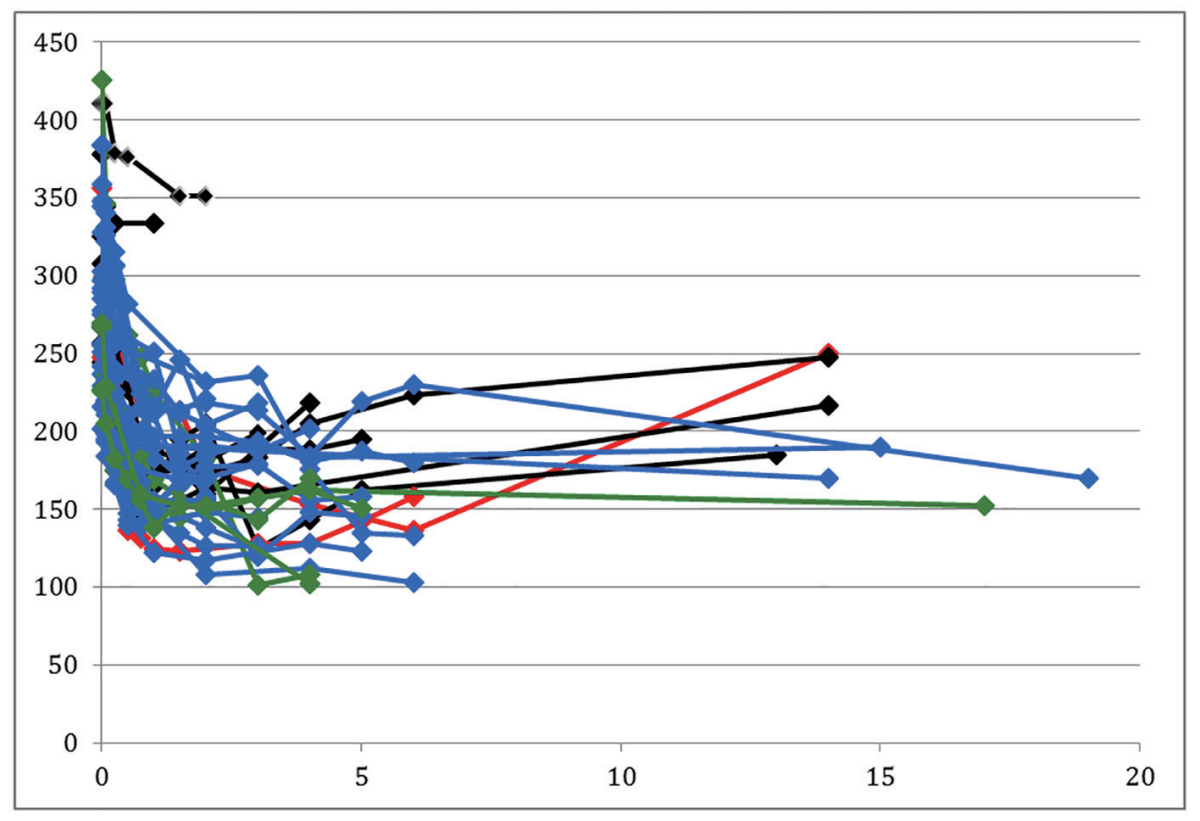

Figure 1. Patient weight changes. Black lines are predicted weight gainers, green lines are those predicted to gain weight but actually lost weight, blue lines are patients predicted to lose weight, and red lines are predicted losers who actually regained.

sification of $32 / 39(82 \%)$. Using linear regression, the model which was described in methods section above, used the cholinergic measure RRI, the adrenergic measures VC and PAR, and the enteric measure EGG. The values from patients were compared with normal control values as a way of constructing the model of disease state. Specific physiologic characteristics of patients who lost weight were compared to those who gained weight. Using this approach, a model that separates patients who gained weight versus those who lost was constructed.

The same model with data at 3 months postoperatively predicted weight gain in $9 / 10(90 \%)$ of patients and weight loss in $24 / 29(83 \%)$ of patients, for a total correct classification of $34 / 39(87 \%)$ (Table 2$)$.

No single discriminant criterion (PAR, VC, RRI, and EGG values) alone predicted weight gain and all 39 patients had at least one abnormal criterion. For instance, of the patients who gained weight, eight had a normal EGG value and only two had abnormal values and of the patients who lost weight 25 had normal EGG values and four had abnormal values.

\section{Discussion}

Obesity has become epidemic in the United States: while in 1985 no state had an obesity prevalence greater than $14 \%$, by 2010 every state had a prevalence of $20 \%$ or more [28]. Data from National Health and Nutrition Examination Survey from 2007 to 2012 show that, in the United States, 39.96\% of men and $29.74 \%$ of women were overweight and $35.04 \%$ of men and $36.84 \%$ of women were obese [29]. Treatment success has been variable, with medications improving weight loss 3-5\% over that of placebo and there has been recognition that long-

Table 2. Prediction of Weight Change by Model

\begin{tabular}{|c|c|c|c|c|}
\hline & Actual weight gain & & Actual weight loss & Total \\
\hline \multicolumn{5}{|l|}{ Using data obtained at baseline } \\
\hline Gainer model predicted weight gain & $8(80 \%)$ & Loser model predicted weight gain & $5(17 \%)$ & 13 \\
\hline Totals & 10 & & 29 & \\
\hline \multicolumn{5}{|c|}{ Overall correct classification rate: $32 / 39(82 \%)$} \\
\hline \multicolumn{5}{|l|}{ Using data 3 months postoperative data } \\
\hline Gainer model predicted weight loss & $1(10 \%)$ & Loser Model Predicted Weight Loss & $24(83 \%)$ & 25 \\
\hline Totals & 10 & & 29 & \\
\hline Overall correct classification rate: $34 /$ & $(87 \%)$ & & & \\
\hline
\end{tabular}


term maintenance of weight loss is difficult [30]. There is debate about how to best define gastric obesity surgery success $[31,32]$; most studies evaluating long-term success have an endpoint of only 2 years [33].

The strength of our study is the longevity of our data, which demonstrates the commonly observed outcome that, post-restrictive gastric surgery, weight gain occurs after the 2 -year endpoint. For instance, only four of our 10 patients with long-term weight gain resumed gaining their weight at 2 years. Conversely, six of the 10 long-term weight gainers began regaining their weight after the 2-year time point, raising doubts that prior studies with a 2 -year endpoint have correctly estimated the long-term success of restrictive surgery.

In this study, while no single measurement of the ANS and ENS predicted weight loss, but clustering the PAR, VC, RRI, and EGG values together as predictor variables using discriminant analysis was able to arrive at the categorical dependent variable of future weight gain and thus develop a model for future validation.

Bariatric surgery research has had variable definitions of success. Our long-term data considered a regaining of 20 pounds or a final weight of more than 175 pounds as a failure. This was done because these particular cut-offs delineated those patients who had on-going post-surgical weight gain from those with continued weight loss, or were maintaining a healthy weight. Our study was limited, as the values for patients' heights were not consistently recorded, since the focus of this study at the time of surgery was patients' actual weight loss.

By incomplete collection of patients' heights, we could not make calculations relying on heights such as ideal body weights and BMI consistently available. Prior authors have utilized patients' heights to normalize weight loss. For instance, in 1982 Reinhold reviewed prior methods of determining success after gastric bypass surgery as well as presenting his own 29 patients 1 year after surgery [34]. The Reinhold criteria have perhaps been misquoted as 50\% excess weight loss (EWL). His paper stratified his results using two different methods, both of which relied on their ideal body weight (IBW). In the first he calculated their percent final excess weight $(\%$ FEW) (the difference of the final weight less and the IBW divided by the IBW). Weight loss after 12 months was determined to be excellent $<25 \% \mathrm{FEW}$, good 26-50\% FEW, fair 51-75\% FEW, poor $76-100 \% \mathrm{FEW}$, and failure $>100 \% \mathrm{FEW}$. The second method Reinhold used was absolute excess final weight (AEFW) being the difference of final weight and IBW. Cut-offs for this method were excellent $<11.4 \mathrm{~kg}$, good $11.5-22.8 \mathrm{~kg}$, fair $22.9-34.2$ $\mathrm{kg}$, poor $34.3-45.5 \mathrm{~kg}$, and failure $>45.7 \mathrm{~kg}$. He also presented that weight regain occurred in seven of his 29 patients, but was most concerned with two patients who regained more than 20 $\mathrm{kg}$ and both had excess intake of alcohol.

Definitions for success after weight loss surgery have emerged which take into consideration the patient's height and have been criticized as misleading because of large variability in patients' initial BMI [32]. It relies on the patient's height to calculate the IBW. The difference from the patient's weight at surgery from the IBW is the excess body weight (EBW) which is used as a denominator for the percentage of excess weight loss $\%$ EWL. Subtracting the current weight from weight at surgery determines the current weight loss (WL). Dividing the
WL by the EBW yields the EWL.

Since a taller height will result in a larger IBW, and eventually a smaller EBW, this smaller denominator will raise the EWL in taller individuals making it a difficult comparison between patients with variable heights and BMIs. Additionally, it is possible for a patient to regain significant weight a few years after the surgery and still have an EWL that is at goal.

The goals for EWL have varied. In the largest series of vertical banded gastroplasty patients, the EWL goal at 10 years was more than $25 \%$ of excess without another operation [20]. In his series, $79.37 \%$ of patients were able to have $>25 \%$ EWL. In the gastric bypass literature, a 50\% EWL has been used as a numerical goal for success. Long-term data from O'Brien and colleagues' restrictive gastric procedure patients which included a large number of patients who were followed beyond 3 years had an EWL around 50\% with a 1\% confidence interval [35]. A recent meta-analysis, which included studies with follow-up time exceeding 3 years, also compared excess weight loss and not actual weight loss [2].

Guidance is lacking on which patients might benefit most from weight loss surgery. A worthwhile goal would be to more accurately exclude patients that will regain weight long-term and select those who will have long-term benefits. Building a model to describe long-term weight gainers and losers is the first step; with greater numbers, the model can be refined, validated and have improved accuracy to provide patients and surgeons with a powerful tool in deciding whom should undergo a surgery.

A second question raised by the data is: if autonomic parameters predicted weight loss, could changing a patient's native values change them from long-term weight gain to continued weight loss? Enteric, adrenergic, and cholinergic measures have previously been shown to improve using gastric electrical stimulation (GES) for gastroparesis [36] and also after pancreas-kidney transplantation $[4,6,9]$. We hypothesize that if a patient's autonomic testing predicts weight gain, then GES might convert the patient's physiology into a long-term weight loss phenotype.

\section{Conclusion}

In conclusion, we observe that long-term vertically banded gastroplasty weight gainers and losers appear to be predicted by a discriminate model based on ANS and ENS evaluation. Further research may help select patients who will benefit most from gastric bypass surgery, and provide insights on how modulation of the ANS using GES can potentially become a weight loss intervention.

\section{Acknowledgments}

The authors would like to thank a number of individuals at the University of Tennessee-Memphis (now University of TN Health Sciences Center): especially Dr. George Cowan, whose patients agreed to be studied and who operated on the original 39 patients; his subsequent colleagues Dr. A. K. Madan and Dr. D. S. Tichansky, for help with follow-up data acquisition; and Dr. 
Sergio Cardoso, who founded the autonomic lab in Memphis and without whom this work could not have been performed. We would also like to thank the staff of the Autonomic Laboratory at University of Tennessee Bowld Hospital, the Department of Surgery at University of Tennessee, Memphis, and the other individuals in Memphis and at the University of Mississippi in Jackson Mississippi who allowed this work, both short- and long-term, to occur. We would also like to thank Catherine McBride at the University of Louisville for help with manuscript preparation, as well as Dr. Guy Brock at the University of Louisville for helpful suggestions in preparing this manuscript.

\section{Consent}

Patient valuation and subsequent follow-up are part of ongoing IRB/ethical approvals at the University of TennesseeMemphis, the University of Mississippi Medical Center, and the University of Louisville Medical Center. For this type of study, formal consent is not required.

\section{Competing Interests}

Drs. Abell, Johnson, and Rashed are named in a patent on certain aspects of the technology listed in this manuscript, and awarded to the University of Mississippi Medical Center.

\section{Funding}

No additional funding was used for this study.

\section{Author Contributions}

$\mathrm{NC}$ : data management, preparation of manuscript. TA and HR: study concept, data acquisition and management, manuscript preparation. WJ: data analysis, manuscript preparation. GC: study concept, data acquisition and management, manuscript preparation. TC, DT, AM and NA: data acquisition and management.

\section{Abbreviations}

PAR: postural adjustment ratio; VC: vasoconstriction; EGG: electrogastrogram; EKG: electrocardiogram; RRI: R-to-R interval; ANS: autonomic nervous system; ENS: enteric nervous system; BMI: body mass index; cpm: cycles per minute; EWL: excess weight loss; IBW: ideal body weight; \%FEW: final excess weight; AEFW: absolute excess final weight

\section{References}

1. DeHaven J, Sherwin R, Hendler R, Felig P. Nitrogen and sodium balance and sympathetic-nervous-system activ- ity in obese subjects treated with a low-calorie protein or mixed diet. N Engl J Med. 1980;302(9):477-482.

2. Puzziferri N, Roshek TB, 3rd, Mayo HG, Gallagher $\mathrm{R}$, Belle $\mathrm{SH}$, Livingston EH. Long-term follow-up after bariatric surgery: a systematic review. JAMA. 2014;312(9):934-942.

3. Horowitz M, Collins PJ, Cook DJ, Harding PE, Shearman DJ. Abnormalities of gastric emptying in obese patients. Int J Obes. 1983;7(5):415-421.

4. Gaber AO, Hathaway DK, Abell T, Cardoso S, Hartwig $\mathrm{MS}$, el Gebely S. Improved autonomic and gastric function in pancreas-kidney vs kidney-alone transplantation contributes to quality of life. Transplant Proc. 1994;26(2):515-516.

5. Gaber AO, Oxley D, Karas J, Cardoso S, Hathaway D, Shokouh-Amiri MH, Jensen SL, et al. Changes in gastric emptying in recipients of successful combined pancreaskidney transplants. Dig Dis. 1991;9(6):437-443.

6. Hathaway D, Abell T, Cardoso S, Hartwig M, Elmer D, Horton J, Lawrence D, et al. Improvement in autonomic function following pancreas-kidney versus kidney-alone transplantation. Transplant Proc. 1993;25(1 Pt 2):13061308.

7. Berthoud HR. Vagal and hormonal gut-brain communication: from satiation to satisfaction. Neurogastroenterol Motil. 2008;20(Suppl 1):64-72.

8. Ikramuddin S, Blackstone RP, Brancatisano A, Toouli J, Shah SN, Wolfe BM, Fujioka K, et al. Effect of reversible intermittent intra-abdominal vagal nerve blockade on morbid obesity: the ReCharge randomized clinical trial. JAMA. 2014;312(9):915-922.

9. Gaber AO, Cardoso S, Pearson S, Abell T, Gaber L, Hathaway D, Alakkad M, et al. Improvement in autonomic function following combined pancreas-kidney transplantation. Transplant Proc. 1991;23(1 Pt 2):1660-1662.

10. Hathaway DK, Abell T, Cardoso S, Hartwig MS, el Gebely $\mathrm{S}$, Gaber AO. Improvement in autonomic and gastric function following pancreas-kidney versus kidney-alone transplantation and the correlation with quality of life. Transplantation. 1994;57(6):816-822.

11. Abell TL, Cowan G, Cardoso S, Familoni B, Defibaugh N. An autonomic and electrogastrographic study of patients with morbid obesity. Gastroenterology. 1990;98(Part 2):A320.

12. Cowan G, Abell TL, Defibaugh N, Cardoso S, Familoni B, Hiler ML. Preliminary autonomic and electrogastrographic studies in preoperative bariatric surgery patients. Obesity Surgery. 1991:112.

13. Abell TL, Cardoso S, Karas J, Familoni J, Cowan G. Long-term followup of patients who have undergone surgery for morbid obesity. In: AGA National Meeting; 1994:A594.

14. Abell TL, Tabbaa M, Rashed H, Cardoso S, Familoni J, Buffington C, Cowan G. Long term follow-up of obesity surgery-autonomic and enteric nervous system predictors at baseline are important. Gastroenterology. 1997;92:1748.

15. Aslam N, Rashed H, Madan AK, Tichansky DS, Cutts T, Abell TL. Long term follow-up of autonomic and enteric 
measures in patients undergoing vertical banded gastropathy for morbid obesity. Neurogastroenterol Motil. 2005:472.

16. Abell TL, Tabbaa M, Rashed H, Cardoso S, Familoni J, Buffington C, Cowan G. Long term follow-up of obesity surgery-autonomic and enteric nervous system predictors at baseline are important. In: DDW; New Orleans, LA; 1998.

17. Aslam N, Rashed H, Madan AK, Tichansky DS, Cutts T, Johnson WD, Abell TL. A multi-component model can predict outcome of restrictive surgery for obesity at baseline and 3 months. In: SSCI; Atlanta, GA; 2006:S1965.

18. Aslam N, Rashed H, Madan AK, Tichansky DS, Cutts T, Johnson WD, Abell TL. A multi-component model can predict outcome of restrictive surgery for obesity at baseline and 3 months. In: DDW; 2006:S1965.

19. Aslam N, Rashed H, Madan AK, Tichansky DS, Cutts T, Johnson WD, Abell TL. A multi-component model can predict outcome of restrictive surgery for obesity at baseline and 3 months. In: IEGGS; 2006:A10.

20. Mason EE, Doherty C, Cullen JJ, Scott D, Rodriguez EM, Maher JW. Vertical gastroplasty: evolution of vertical banded gastroplasty. World J Surg. 1998;22(9):919-924.

21. Lin YH, Lee WJ, Ser KH, Chen SC, Chen JC. 15-year follow-up of vertical banded gastroplasty: comparison with other restrictive procedures. Surg Endosc. 2016;30(2):489-494.

22. Abell TL, Cardoso S, Schwartzbaum J, Familoni B, Wilson R, Massie D. Diabetic gastroparesis is associated with an abnormality in sympathetic innervation. Eur J Gastroenterol Hepatol. 1994;6:241-247.

23. Kim CH, Hanson RB, Abell TL, Malagelada JR. Effect of inhibition of prostaglandin synthesis on epinephrineinduced gastroduodenal electromechanical changes in humans. Mayo Clin Proc. 1989;64(2):149-157.

24. Aggarwal A, Cutts TF, Abell TL, Cardoso S, Familoni B, Bremer J, Karas J. Predominant symptoms in irritable bowel syndrome correlate with specific autonomic nervous system abnormalities. Gastroenterology. 1994;106(4):945-950.

25. Rashed H, Abell TL, Familoni BO, Cardoso S. Autonomic function in cyclic vomiting syndrome and classic migraine. Dig Dis Sci. 1999;44(8 Suppl):74S-78S.
26. Aslam N, Kedar A, Nagarajarao HS, Reddy K, Rashed H, Cutts T, Riely C, et al. Serum catecholamines and dysautonomia in diabetic gastroparesis and liver cirrhosis. Am J Med Sci. 2015;350(2):81-86.

27. Abell TL, Malagelada JR. Electrogastrography. Current assessment and future perspectives. Dig Dis Sci. 1988;33(8):982-992.

28. Bauer UE, Briss PA, Goodman RA, Bowman BA. Prevention of chronic disease in the 21st century: elimination of the leading preventable causes of premature death and disability in the USA. Lancet. 2014;384(9937):45-52.

29. Yang L, Colditz GA. Prevalence of Overweight and Obesity in the United States, 2007-2012. JAMA Intern Med. 2015;175(8):1412-1413.

30. Eckel RH. Clinical practice. Nonsurgical management of obesity in adults. N Engl J Med. 2008;358(18):19411950.

31. Hatoum IJ, Kaplan LM. Advantages of percent weight loss as a method of reporting weight loss after Roux-en-Y gastric bypass. Obesity (Silver Spring). 2013;21(8):15191525.

32. van de Laar A, de Caluwe L, Dillemans B. Relative outcome measures for bariatric surgery. Evidence against excess weight loss and excess body mass index loss from a series of laparoscopic Roux-en-Y gastric bypass patients. Obes Surg. 2011;21(6):763-767.

33. Buchwald H, Avidor Y, Braunwald E, Jensen MD, Pories W, Fahrbach K, Schoelles K. Bariatric surgery: a systematic review and meta-analysis. JAMA. 2004;292(14):17241737.

34. Reinhold RB. Critical analysis of long term weight loss following gastric bypass. Surg Gynecol Obstet. 1982;155(3):385-394.

35. O'Brien PE, MacDonald L, Anderson M, Brennan L, Brown WA. Long-term outcomes after bariatric surgery: fifteen-year follow-up of adjustable gastric banding and a systematic review of the bariatric surgical literature. Ann Surg. 2013;257(1):87-94.

36. Luo J, Rashed HM, Eaton P, Voeller G, Familoni J, Abell TL. Long term treatment at gastric electrical stimulation is associated with autonomic and enteric nervous system changes [ABSTRACT]. Gastroenterology. 2000;118:A1187. 